\title{
AbobotulinumtoxinA Versus OnabotulinumtoxinA in Adults with Upper Limb Spasticity: A Randomized, Double-Blind, Crossover Study Protocol
}

\author{
Alberto Esquenazi · Ziyad Ayyoub · Monica Verduzco-Gutierrez • \\ Pascal Maisonobe · James Otto · Atul T. Patel
}

Received: July 14, 2021 / Accepted: August 13, 2021 / Published online: September 25, 2021

(C) The Author(s) 2021

\section{ABSTRACT}

Introduction: The safety and efficacy of both abobotulinumtoxinA and onabotulinumtoxinA for upper limb spasticity are well established, but head-to-head comparisons are lacking.

Methods: DIRECTION is an international, randomized, double-blind, crossover study comparing the safety and efficacy of abobotulinumtoxinA with onabotulinumtoxinA in the management of upper limb spasticity at doses at or near maximum recommended in product labelling. Participants (18-75 years) will be randomized (1:1) to either one cycle of

Supplementary Information The online version contains supplementary material available at https:// doi.org/10.1007/s12325-021-01896-3.

A. Esquenazi $(\square)$

MossRehab \& Albert Einstein Medical Center, Elkins

Park, PA 19027, USA

e-mail: aesquena@einstein.edu

Z. Ayyoub

Rancho Los Amigos National Rehabilitation Center, Downey, CA 90242, USA

M. Verduzco-Gutierrez

Joe R. and Teresa Lozano Long School of Medicine, The University of Texas Health Science Center at

San Antonio, San Antonio, TX 78229, USA

P. Maisonobe

Ipsen, 92100 Boulogne-Billancourt, France
abobotulinumtoxinA (900U) followed by onabotulinumtoxinA (360U) or vice versa. To maintain blinding, a fixed volume $(3.6 \mathrm{ml})$ will be injected into the target upper limb muscles (four wrist and finger flexors and biceps brachii). The second treatment cycle will begin at Week 12 if retreatment criteria are fulfilled, and if not, they will be reassessed every 4 weeks until they meet retreatment parameters.

Planned Outcomes: The primary hypothesis is that there is comparable safety between products; non-inferiority will be tested based on treatment-emergent adverse event (TEAE) rates from injection to Week 12. A secondary hypothesis is that abobotulinumtoxinA has longer duration of effect than onabotulinumtoxinA. This hypothesis will be tested with secondary efficacy endpoints, including injec-

J. Otto

Ipsen, Cambridge, MA 02142, USA

\section{A. T. Patel}

Kansas Institute of Research, Overland Park, KS 66211, USA

Z. Ayyoub

David Geffen School of Medicine, University of

California, Los Angeles, Los Angeles, CA 90095, USA

Z. Ayyoub

Western University of Health Sciences, Pomona, CA 91766, USA 
tion cycle duration, Modified Ashworth Scale, Disability Assessment Scale and Physician Global Assessment.

Trial Registration: EudraCT (http://eudract. ema.europa.eu): 2021-000161-32 and Clinicaltrials.gov NCT04936542.

Keywords: AbobotulinumtoxinA; Botulinum toxin; Clinical trial; Spasticity; OnabotulinumtoxinA; Upper limb

\section{Key Summary Points}

DIRECTION will be the first randomized double-blind, crossover study to directly compare the safety and efficacy of abobotulinumtoxinA and onabotulinumtoxinA in adult patients with upper limb spasticity.

The study compares the two products using doses at or near the maximum recommended in the product labelling.

The primary hypothesis is that the safety profiles of both products are comparable, and non-inferiority will be tested based on treatment-emergent adverse event rates from injection to Week 12.

A secondary hypothesis is that abobotulinumtoxinA has a different duration of effect than onabotulinumtoxinA, and this hypothesis will be based on superiority tests associated with secondary efficacy endpoints analyses, using a hierarchical approach.

\section{DIGITAL FEATURES}

This article is published with digital features, including a video abstract, to facilitate understanding of the article. To view digital features for this article go to https://doi.org/10.6084/ m9.figshare.15164883.

\section{INTRODUCTION}

Adult patients with central nervous system injury often present with limb spasticity. Because upper limb function is essential for many daily living tasks, impairment of arm use contributes to a reduction of the quality of life as well as reduced independence [1]. If untreated, a vicious cycle occurs in which affected muscle groups lead to an abnormal limb posture, resulting in soft tissue shortening and further biomechanical changes in the contracted limb. This in turn prevents muscle lengthening and perpetuates further hypertonicity [2]. There are many anti-spasticity treatment options available, and, while oral systemic treatments are often used to treat spasticity, their lack of specificity and association with serious adverse effects when used at therapeutic doses limits their usefulness. Local intramuscular injection of botulinum toxin type A (BoNT-A) is an established, first line, well-tolerated treatment option supported by a strong body of Level I evidence in the management of upper limb spasticity [2-4].

All BoNT-A products contain the same botulinum neurotoxin, derived from the Hall strain of Clostridium botulinum, but differ in their exact molecular composition, processing and excipients [5]. Due to their distinct formulations and methods of production, each BoNTA product has its own unique set of characteristics, and as such, the dosing safety and efficacy characteristics must be judged separately. In addition, it is important to consider that all BoNT-A products are biologic preparations, and their activity is measured in units (U). These units of biologic activity are assessed using different assay methods, and hence they cannot be considered equivalent [6]. Thus, the units of one BoNT-A cannot be compared to or converted into units of any other BoNT-A products. Several external factors also influence the clinical profile of BoNT-A when injected into muscles. These include the anatomy of the target areas to be injected, the size of the affected 
muscles (e.g., large or small), degree of spasticity and injection technique (e.g., volume of injection, number of sites per muscle, and guidance use).

The safety and efficacy of abobotulinumtoxinA (aboBoNT-A) [7-10] and onabotulinumtoxinA (onaBoNT-A) [11-13] in upper limb spasticity have been investigated and demonstrated in clinical trials versus placebo. However, while both products alleviate clinical symptoms of spasticity compared to placebo, their safety and efficacy have never been compared in a blinded prospective study. Recent observational studies suggest differences in the intervals needed between injections (with patients on aboBoNT-A having longer intervals between injections than onaBoNT-A) [14], but such observations require prospective confirmation. Such information on the potential differences of each product is of great importance to inform treatment decisions. For example, a switch from one BoNT-A to the other might be necessary as per physician's decision based on patient medical needs to optimize treatment outcomes or based on hospital/insurance budgetary directives for financial considerations $[15,16]$. Moreover, recent data from a patient survey in spasticity indicate that $>80 \%$ patients with spasticity are living with significant symptom re-emergence between injections [17], and an understanding of the duration of benefit will be important in ensuring this 'rollercoaster' of symptoms is reduced or avoided.

An important methodologic consideration in comparative studies of BoNT-A products is the choice of doses. The US Food and Drug Administration (FDA) and European agencies have determined that each BoNT-A is unique and noninterchangeable and its dosing is specific [18]. Uncertainty regarding dose conversion ratios arises from inconsistent literature which includes wide variation in the patient populations, clinical settings, study designs, outcomes assessed, and injection techniques studied. This variability has resulted in a wide range of dosing ratios between BoNT-A products, with conversion factors ranging from $1: 1.67$ to $1: 11$ for onaBoNT-A: aboBoNT-A $[19,20]$. More recently, several authors have concluded that a single fixed ratio cannot be applied to all individuals [19, 21, 22]. Indeed, studies using a predefined dose ratio may be particularly misleading because they rely on the hypothesis that the defined doses of the different formulations are equivalent (and frequently fail to prove this hypothesis). A more practical way to compare doses is to utilize doses at or near the maximum recommended in the product labelling. Each product summary of characteristics/prescribing information is based on data from clinical trials which have tested the safety and efficacy of prespecified doses or dose ranges for each product in the targeted patient populations.

The DIRECTION study aims to compare the safety and efficacy of aboBoNT-A and onaBoNTA for upper limb spasticity when used at doses at or near maximum recommended in the US product labelling. To ensure full comparability in a double-blind setting, we have chosen to focus on the same pattern of spasticity (involving the finger and wrist flexors and biceps brachii) and have standardized the injection approach. The primary hypothesis is that the safety profiles of both products are comparable, and non-inferiority will be tested based on treatment-emergent adverse event (TEAE) rates from injection to week 12. A secondary hypothesis is that aboBoNT-A has different duration of effect than onaBoNT-A, and this hypothesis will be based on superiority tests associated with secondary efficacy endpoint analyses, using a hierarchical approach.

\section{METHODS}

\section{Study Design}

DIRECTION is an international, randomized, double-blind, crossover, post-marketing study comparing the clinical safety and efficacy of aboBoNT-A with onaBoNT-A in the management of adult upper limb spasticity. For an overview of the protocol, see the video in the online/HTML version of the manuscript or follow the digital features link under the abstract. This study has been registered on the EudraCT (2021-000161-32) and clinicaltrials.gov (NCT04936542) databases. Study protocols will 
be approved at each site. This study will be conducted in accordance with the Helsinki Declaration of 1964 and its later amendments and the ICH Good Clinical Practice Guidelines.

Participants will be randomized 1:1 to one of two sequences of treatment:

1. Participants will receive one cycle of aboBoNT-A (900 Units) followed by one cycle of onaBoNT-A (360 U) in the same selected overactive upper limb muscles using injection guidance techniques.

2. Participants will receive one cycle of onaBoNT-A (360 Units) followed by one cycle of aboBoNT-A (900 U) in the same selected overactive upper limb muscles using injection guidance techniques.

The second BoNT-A injection will be given at week 12 at the earliest if retreatment criteria are fulfilled. If not, participants will be reassessed every 4 weeks (at week 16, week 20 and week 24) until retreatment criteria are fulfilled, at which point the second BoNT-A will be administered. If retreatment criteria are still not fulfilled by week 24 of Cycle 1, the participant will be withdrawn from the study and "no clinical indication for reinjection" documented as the reason for withdrawal.

Visits for each injection cycle will include the injection visit, week 1 , week 4 , week 10 , week 12 and additional visits at week 16 , week 20 and week 24 as required (Fig. 1). In exceptional circumstances, where a scheduled visit cannot be performed (e.g., due to COVID 19), data on AEs and retreatment criteria may be collected by telephone. The End of Study (EOS) for each participant will be when the decision is made to retreat or at week 24 of cycle 2 , whichever occurs first. The participants will be followed up for safety and efficacy, including quality of life (QoL), until their EOS or early withdrawal (EW) visit. Participants withdrawing early from the study will not be replaced.

\section{Sample Selection}

Eligible participants (aged 18-75 years of age inclusive) must have had upper limb spasticity for $\geq 3$ months, with treatment of only one upper limb being necessary for the duration of the study. Due to differences in label indications, sites in the US and France are allowed to recruit patients with upper limb spasticity due to any etiology, while Canadian sites are restricted to recruiting only those with upper limb spasticity due to stroke. Eligible participants must show a pattern of upper limb spasticity that requires BoNT-A injection in all of the following muscles: flexor carpi radialis, flexor carpi ulnaris, flexor digitorum profundus, flexor digitorum superficialis and biceps brachii. They must have a modified Ashworth Scale (MAS) score [23] $\geq 2$ at elbow, wrist and finger flexors and a Disability Assessment Scale (DAS) score $[11] \geq 2$ on the principal target of treatment (one of four functional domains: dressing, hygiene, limb position and pain). Patients may be naïve to BoNT-A treatment or previously treated (with a treatment interval of $\geq 12$ weeks since the last injection). All participants should be stable for $\geq 3$ months prior to study entry in terms of any concomitant oral antispasticity, anticoagulant and/or anticholinergic medications and for $\geq 1$ month prior to study entry in terms of any occupational therapy and/or physiotherapy treatment and must be considered by the investigator likely to remain stable for the duration of the study. All participants must be able to provide written informed consent.

Key exclusion criteria include any major limitations in the passive range of motion in the paretic upper limb and any major neurologic impairment (other than limb paresis) that could negatively affect functional performance. Participants clinically requiring injection into any upper limb muscles other than the five muscles, or requiring injection into both arms or any lower limb within the timeframe of the study, will be excluded. Other exclusion criteria include known hypersensitivity to any BoNT product or excipients or cow's milk protein (casein), infection at the proposed injection site(s), known peripheral motor neuropathic diseases, amyotrophic lateral sclerosis or neuromuscular junction disorders (e.g., myasthenia gravis or Lambert-Eaton syndrome), pregnancy and any medical condition (including dysphagia or breathing difficulties/compromised 


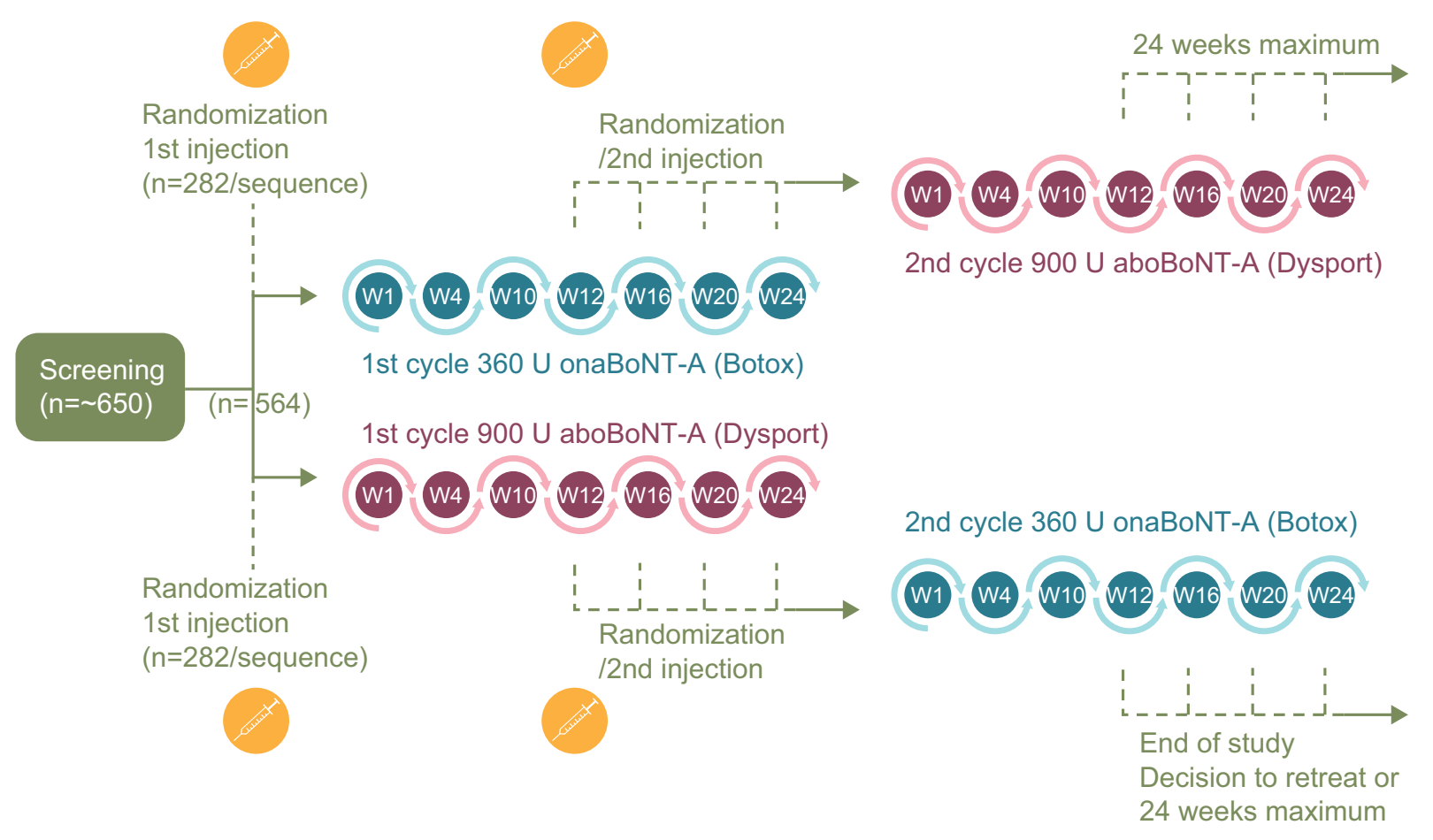

Fig. 1 Study design. aboBoNT- $A$ abobotulinumtoxinA, onaBoNT- $A$ onabotulinumtoxinA, $W$ week

respiratory function) that, in the opinion of the investigator, might jeopardize the participant's safety.

\section{Sample Size Calculation, Recruitment and Randomization}

Based on previous studies [7], it is estimated that $44 \%$ of participants will experience a treatment-emergent adverse event (TEAE) between injection and week 12. Assuming a non-inferiority margin of 5\%, a nuisance parameter of $20 \%$ and a $10 \%$ drop-out rate, a sample size of 564 participants is estimated to give $80 \%$ power to show no difference between the two treatment groups.

Participants will be recruited from the investigators' clinical practice at approximately 90 study sites in the USA, Canada and France (countries which share similar labels for aboBoNT-A and onaBoNT-A to allow for the planned 'on-label' comparison). A total of approximately 650 participants will be screened to give 564 eligible participants (282 participants per sequence). Assignment to study intervention (randomization) will be stratified based on the BoNT-A status (BoNT-A previously treated for upper limb spasticity or naïve to BoNT-A treatment). Randomization will be managed by an Interactive Web Response System. After eligibility is confirmed, at baseline, participants will be assigned a randomization number and to the associated treatment sequence, in sequential order within each center (and within each level of strata).

\section{Treatment}

Participants will be treated in accordance with the approved local labels of aboBoNT-A and onaBoNT-A, which contain further details of the products as well as the requirements of the protocol. Randomized participants will receive either one cycle of aboBoNT-A (900 U) followed by one cycle of onaBoNT-A (360 U) or vice versa. Randomization will be performed using a computer-generated system, and participants will be assigned using an interactive web response system (IWRS). To maintain study blinding, study medications will be 
reconstituted to a fixed total volume $(3.6 \mathrm{ml})$ by separate study staff, and the blinded injector will inject the target upper limb muscles in a fixed volume $(0.5 \mathrm{ml}$ into each wrist and finger flexor and $1.6 \mathrm{ml}$ into biceps brachii) (Fig. 2). The biceps brachii must be treated with two injections, with a maximum single injection volume of $1.0 \mathrm{ml}$. The use of injection guidance techniques to target the injection sites is mandatory in this study. Guidance techniques can be electromyography and/or electrical stimulation for each muscle, with additional ultrasound if needed. The same guidance technique must be used in both cycles. Because of the possibility of site injection stiffness and pain, any intramuscular vaccination to the shoulder (e.g., COVID 19) should be administered in the arm contralateral to the study medication.

Participants must meet all of the following criteria to be eligible for retreatment:
2. MAS score in the primary target muscle group (PTMG) has returned to baseline (i.e., no improvement relative to Day 1 before Cycle 1 injection as indicated by decreased MAS score);

3. No unacceptable safety risk for the participant to receive the next treatment cycle, based on the investigator's judgment;

4. No COVID 19 vaccine injection received within 7 days before the second planned study intervention injection or planned/likely to be received within 7 days after the second planned study intervention injection.

Retreatment may be at week 12 (earliest retreatment timepoint), week 16 , week 20 or week 24 (latest retreatment timepoint). However, retreatment criteria will also be assessed at week 10 even though reinjection cannot take place until Week 12 at the earliest.

1. Decided per the investigator's clinical judgment;

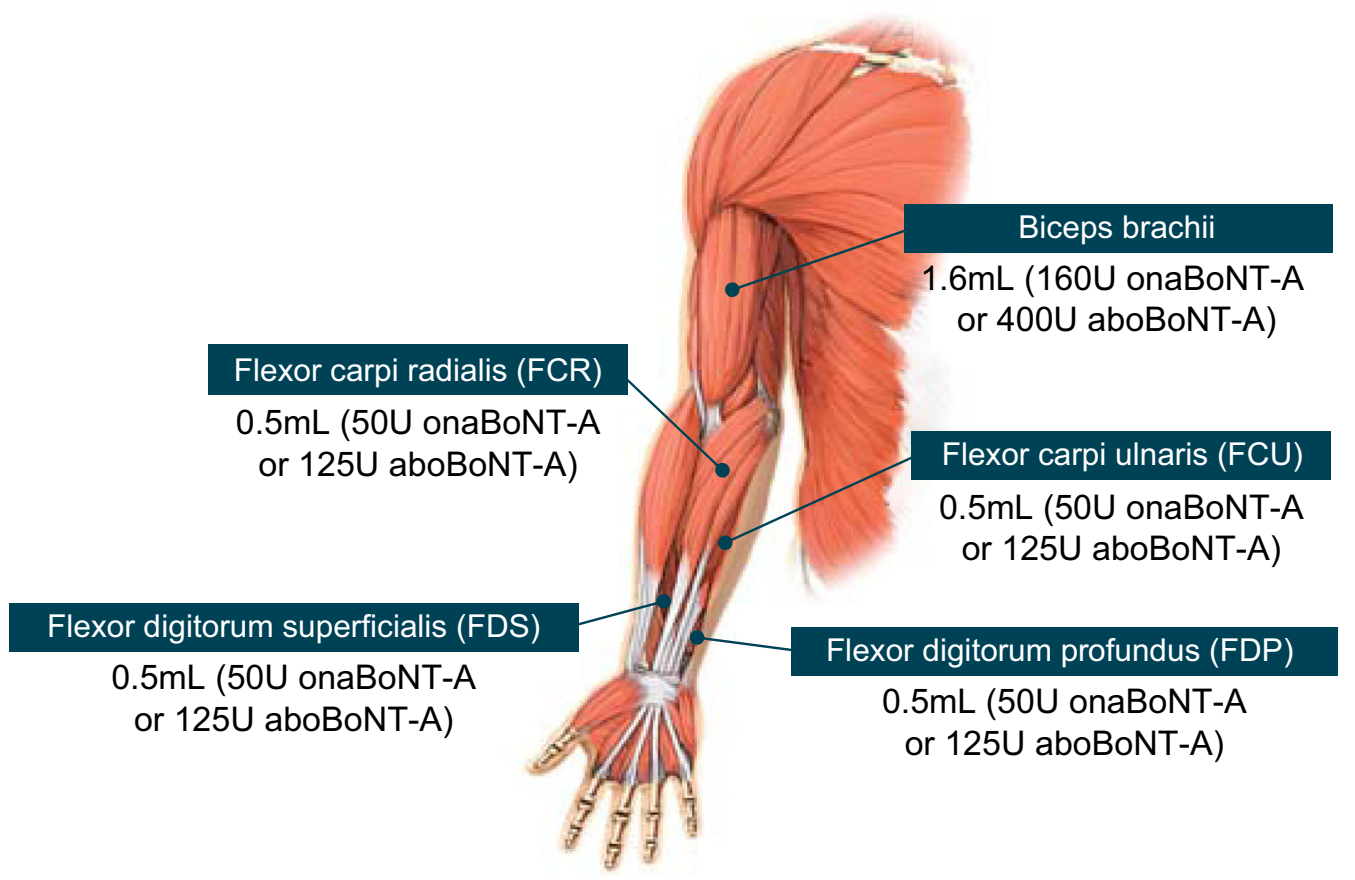

Fig. 2 Injection scheme. aboBoNT-A abobotulinumtoxinA, onaBoNT- $A$ onabotulinumtoxinA 


\section{Measurements and Planned Outcomes}

Each participant will have the same injector for both cycles and the same assessor for all their assessments throughout the study. Safety will be closely monitored during the study. Type, severity, seriousness, duration, reversibility and outcome of treatment-emergent adverse events (TEAEs) and adverse events of special interest (AESIs including adverse events indicative of remote spread), as well as TEAEs leading to withdrawal, will be followed throughout the study.

Efficacy assessments will include:

- Duration of response defined (per cycle) as the time between the injection and the first visit when retreatment criteria are met (from week 10 visit) or the time between injection and study withdrawal if the participant discontinues from the study without meeting retreatment criteria.

- MAS based on the PTMG as selected by the investigators at screening as the muscle group with the highest MAS score. The PTMG is to remain the same throughout the study.

- DAS based on the principal target of treatment as defined at screening based on one of the four DAS domains (i.e., dressing, hygiene, limb position and pain).

- Physician global assessment (PGA) of response, which will be assessed on a 9-point rating scale ( -4 : markedly worse, -3 : much worse, -2 : worse, -1 : slightly worse, 0 : no change, +1 : slightly improved, +2 : improved, +3 : much improved and +4 : markedly improved) by asking the investigator the following question: 'how would you rate the response to treatment in the participant's upper limb since the last injection?'

- Quality of life assessments will be performed using SF-12 [24] and SQoL-6D [25] questionnaires at baseline, week 4 , week 12 and the end of each cycle (which may be week 16, week 20 and week 24 if these visits are performed).

\section{Data Analysis}

For the primary objective, a generalized linear mixed model approach will be used to estimate the difference in TEAE rate between the two treatment groups while accounting for withinparticipant correlations arising from the crossover design. The fitted model will include fixed effects for treatment and cycle and a random effect associated with the participant. The treatment by cycle interaction (carryover effect) will be tested: if the associated $p$-value is $>0.10$, it will be considered absent or negligible and will not be included in the final model to provide estimates. The estimated difference in TEAE rate (aboBoNT-A-onaBoNT A) and associated two-sided 95\% confidence interval (CI) (or one-sided $97.5 \% \mathrm{CI}$ ) will be provided: the non-inferiority of aboBoNT-A versus onaBoNTA in terms of safety profile will be demonstrated if the upper bound of this CI is $<5 \%$ in the Per Protocol Set for safety analyses.

A hierarchical approach will be used for analysis of primary and secondary endpoints. If non-inferiority is demonstrated for the primary endpoint, first the superiority of aboBoNT-A over onaBoNT-A will be tested based on the key secondary endpoint, i.e., the duration of response based on retreatment criteria. If superiority of aboBoNT-A over onaBoNT-A is demonstrated, then MAS/DAS/PGA at week 12 will be tested at the same time. If positive, then the corresponding endpoints at week 10 will be tested.

Efficacy endpoints will compare the treatment groups using a mixed effects model with cycle and treatment as fixed effects and participant as random effect. To preserve an overall type I error rate of 0.05 (one-sided 0.025) for the primary key and secondary endpoints, a multiplicity adjustment involving Bonferroni adjustment and serial gatekeeping procedure with pre-specified hierarchical order will be performed. Each hypothesis will be formally tested only if the previous hypothesis in the prespecified hierarchical order is tested significantly. If a test is not significant, the serial gatekeeping procedure will stop (the result of the test as well as the next tests of the sequence cannot be claimed significant). 


\section{STRENGTHS AND LIMITATIONS}

DIRECTION will be the first study to directly compare the safety and efficacy of aboBoNT-A and onaBoNT-A in adult patients with upper limb spasticity. While both products are also approved for the management of lower-limb spasticity, we chose to focus on the upper limb since (1) it was the first approved adult spasticity indication for both products and (2) the upper limb appeared to allow for the highest treatment effects versus placebo in prior randomized clinical trials (both products) [7, 11]. We compare the two products with the widest worldwide availability; however, other commercial BoNT-A products are available. While the dosing pattern is limited in this study (e.g., excludes patients requiring treatment of the brachialis and pronator teres), it is a treatment pattern commonly encountered in clinical practice [26]. In addition, it conforms to muscles common to both toxin product labels and thus allows for a double-blinded study design.

The primary hypothesis is that there is comparable safety between products and, as such, the study is powered for a non-inferiority design. The secondary hypothesis is that while there is a similar efficacy profile between both products at peak effect (week 4), aboBoNT-A has a longer duration of effect than onaBoNT-A (based on prior observational data [14]). This hypothesis will be associated with a conservative hierarchical secondary efficacy endpoint analysis based on superiority tests, with Bonferroni adjustments for multiplicity. Of note, this will be the first randomized, controlled study of spasticity to prospectively consider duration of BoNT-A effect as part of the effectiveness construct. Whereas the pivotal studies for aboBoNT-A allowed flexibility of reinjection schedules $[7,8,27]$, the limited 12-week study duration for the onaBoNT-A studies prevents even indirect comparisons. Our study design follows patients over the course of two injection cycles and allows flexibility to measure when patient outcomes return to baseline and the crossover study design allows for direct intrasubject comparisons. Although it is possible a patient may meet retreatment criteria in between reassessment visits, we included an assessment at week 10 to detect an early warning of effect and designed the 4-weekly intervals after week 12 to provide balance between the need for flexibility and trial burden on the patient, carer and investigator sites.

While the fixed dosing of both products does not allow for tailored dosing to the individual patient, it was chosen to ensure fair comparability between the two products and is reflective of routine clinical practice because the doses are in full alignment with the prescribing information for both products in the countries where the study is performed. Whereas other studies have imposed presumed conversion ratios between dosing $[19,20]$, our dosing is entirely based on the approved prescribing information for both products, with the assumption that approval is based on optimal dosing. This may be especially important as surveys suggest that both onaBoNT-A and aboBoNT-A are often underdosed in real-world clinical practice [28]. It is important to highlight our decision making in dosing because the resultant aboBoNT-A:onaBoNT-A dosing ratio of 2.5:1 may not apply to other muscles and other indications, and we do not advocate for a standard conversion ratio.

\section{ETHICS AND DISSEMINATION}

This study will be conducted in accordance with the Declaration of Helsinki and Council for International Organizations of Medical Sciences (CIOMS) International Ethical Guidelines and International Conference on Harmonization (ICH) Good Clinical Practice (GCP) Guidelines. The protocol and informed consent forms will be reviewed and approved by the sponsor and the applicable institutional review boards/ethical committees with respect to scientific content and compliance with applicable research and human subjects regulations. Study results will be released to the participating physicians, patients and the general medical community in a peer-reviewed paper. 


\section{CONCLUSIONS}

Using clinically relevant and approved dosing, the DIRECTION study will be the first to prospectively compare abobotulinumtoxinA with onabotulinumtoxinA in upper limb spasticity to allow informed decisions for care optimization.

\section{ACKNOWLEDGEMENTS}

Funding. The DIRECTION study is funded by Ipsen, who employs two of the authors (Pascal Maisonobe and James Otto), procured medical writing support and funded the journal's Rapid Service and Open Access Fees. Pascal Maisonobe and James Otto were involved in the study design and writing of this manuscript.

Medical Writing Support. The authors thank Anita Chadha-Patel, PhD, of ACP Clinical Communications Ltd (Hertfordshire, UK) for providing medical writing support, which was funded by Ipsen (Paris, France) in accordance with Good Publication Practice guidelines.

Authorship. All named authors meet the International Committee of Medical Journal Editors (ICMJE) criteria for authorship for this article, take responsibility for the integrity of the work as a whole, and have given their approval for this version to be published.

Authors' Contributions. Alberto Esquenazi, Ziyad Ayyoub, Monica Verduzco-Gutierrez and Atul T. Patel are investigators in the study and sit on the study steering committee. Pascal Maisonobe is the study statistician and James Otto was involved in the study design and is involved in study conduct. Alberto Esquenazi and James Otto contributed to the first draft equally, and all other authors provided critical review. All authors approved the final version of the submitted manuscript.

Prior Presentation. This protocol has been presented at the International Society of
Physical and Rehabilitation Medicine (ISPRM) Virtual Congress, 12-15 June 2021.

Disclosures. Alberto Esquenazi, Ziyad Ayyoub, Monica Verduzco-Gutierrez and Atul Patel are investigators in the DIRECTION study, and report consultancy for Ipsen. In addition, Alberto Esquenazi reports research funding from Ipsen, Allergan/AbbVie and Merz, and consultancy for Allergan/AbbVie and Shionogi. Ziyad Ayyoub reports consultancy for Ipsen. Monica Verduzco-Gutierrez reports consultancy for Ipsen, Allergan/AbbVie and Merz. Atul Patel reports grants for clinical trials with Allergan/ AbbVie and Revance and speaker fees for Allergan/AbbVie and Ipsen. Pascal Maisonobe and James Otto are employed by Ipsen.

Compliance with Ethics Guidelines. This study will be conducted in accordance with the Declaration of Helsinki and Council for International Organizations of Medical Sciences (CIOMS) International Ethical Guidelines and International Conference on Harmonization (ICH) Good Clinical Practice (GCP) Guidelines. The protocol and informed consent forms will be reviewed and approved by the sponsor and the applicable institutional review boards/ethical committees with respect to scientific content and compliance with applicable research and human subjects regulations. All participants must be able to provide written informed consent.

Data Availability. Protocol details are also available at clinical trials.gov: https:// clinicaltrials.gov/ct2/show/NCT04936542.

Open Access. This article is licensed under a Creative Commons Attribution-NonCommercial 4.0 International License, which permits any non-commercial use, sharing, adaptation, distribution and reproduction in any medium or format, as long as you give appropriate credit to the original author(s) and the source, provide a link to the Creative Commons licence, and indicate if changes were made. The images or other third party material in this article are included in the article's Creative Commons licence, unless indicated otherwise in a credit 
line to the material. If material is not included in the article's Creative Commons licence and your intended use is not permitted by statutory regulation or exceeds the permitted use, you will need to obtain permission directly from the copyright holder. To view a copy of this licence, visit http://creativecommons.org/licenses/by$\mathrm{nc} / 4.0 /$.

\section{REFERENCES}

1. Doan QV, Brashear A, Gillard PJ, Varon SF, Vandenburgh AM, Turkel CC, et al. Relationship between disability and health-related quality of life and caregiver burden in patients with upper limb poststroke spasticity. PM\&R. 2012;4(1):4-10.

2. Royal College of Physicians (UK). Spasticity in adults: management using botulinum toxin. National guidelines 2018. https://www.rcplondon. ac.uk/guidelines-policy/spasticity-adultsmanagement-using-botulinum-toxin. Last accessed Jul 2021.

3. Simpson DM, Hallett M, Ashman EJ. Practice guideline update summary: Botulinum neurotoxin for the treatment of blepharospasm, cervical dystonia, adult spasticity, and headache. Neurology. 2016;86:1-9.

4. Esquenazi A, Albanese A, Chancellor MB, Elovic E, Segal KR, Simpson DM, et al. Evidence-based review and assessment of botulinum neurotoxin for the treatment of adult spasticity in the upper motor neuron syndrome. Toxicon. 2013;67:115-28.

5. Bigalke H. Properties of pharmaceutical products of botulinum neurotoxins. In: Jankovic J, Albanese A, Atassi MZ, Dolly JO, Hallett M, Mayer NH, editors. Botulinum toxin: therapeutic clinical practice and science. Philadelphia: Saunders Elsevier; 2009. p. 389-97.

6. Hambleton P, Pickett AM. Potency equivalence of botulinum toxin preparations. J R Soc Med. 1994;87(11):719.

7. Gracies JM, Brashear A, Jech R, McAllister P, Banach M, Valkovic P, et al. Safety and efficacy of abobotulinumtoxinA for hemiparesis in adults with upper limb spasticity after stroke or traumatic brain injury: a double-blind randomised controlled trial. Lancet Neurol. 2015;14(10):992-1001.

8. Gracies JM, O’Dell M, Vecchio M, Hedera P, Kocer S, Rudzinska-Bar $M$, et al. Effects of repeated
abobotulinumtoxinA injections in upper limb spasticity. Muscle Nerve. 2018;57(2):245-54.

9. McCrory P, Turner-Stokes L, Baguley IJ, De Graaff S, Katrak P, Sandanam J, et al. Botulinum toxin A for treatment of upper limb spasticity following stroke: a multi-centre randomized placebo-controlled study of the effects on quality of life and other person-centred outcomes. J Rehabil Med. 2009;41(7):536-44.

10. Rosales RL, Balcaitiene J, Berard H, Maisonobe P, Goh KJ, Kumthornthip W, et al. Early abobotulinumtoxina (Dysport) in post-stroke adult upper limb spasticity: ONTIME pilot study. Toxins. 2018;10(7):253.

11. Brashear A, Gordon MF, Elovic E, Kassicieh VD, Marciniak C, Do M, et al. Intramuscular injection of botulinum toxin for the treatment of wrist and finger spasticity after a stroke. N Engl J Med. 2002;347(6):395-400.

12. Simpson DM, Gracies JM, Yablon SA, Barbano R, Brashear A. Botulinum neurotoxin versus tizanidine in upper limb spasticity: a placebo-controlled study. J Neurol Neurosurg Psychiatry. 2009;80(4):380-5.

13. Marciniak CM, Harvey RL, Gagnon CM, Duraski SA, Denby FA, McCarty S, et al. Does botulinum toxin type A decrease pain and lessen disability in hemiplegic survivors of stroke with shoulder pain and spasticity?: a randomized, double-blind, placebocontrolled trial. Am J Phys Med Rehabil. 2012;91(12):1007-19.

14. Turner-Stokes L, Jacinto J, Fheodoroff K, Brashear A, Maisonobe P, Lysandropoulos A, et al. Longitudinal goal attainment with integrated upper limb spasticity management including repeat injections of botulinum toxin A: findings from the prospective, observational upper limb international spasticity (ULIS-III) cohort study. J Rehabil Med. 2021;53(2): jrm00157.

15. Grosset DG, Tyrrell EG, Grosset KA. Switch from abobotulinumtoxinA (Dysport) to incobotulinumtoxinA (Xeomin) botulinum toxin formulation: a review of 257 cases. J Rehabil Med. 2015;47(2): 183-6.

16. Dursun N, Akarsu M, Gokbel T, Akyuz M, Karacan C, Dursun E. Switching from onabotulinumtoxin-A to abobotulinumtoxin-A in children with cerebral palsy treated for spasticity: a retrospective safety and efficacy evaluation. J Rehabil Med. 2019;51(5): 390-4.

17. Jacinto J, Varriale P, Pain E, Lysandropoulos A, Esquenazi A. Patient perspectives on the therapeutic profile of botulinum neurotoxin type $A$ in spasticity. Front Neurol. 2020;11:388. 
18. Brin MF, James C, Maltman J. Botulinum toxin type A products are not interchangeable: a review of the evidence. Biologics. 2014;8:227-41.

19. Zhang Y, Coulton K, Rosales R, Moore AP, Plested M. Do clinically equivalent dosing ratios exist between different formulations of botulinum toxinA in the treatment of adult spasticity, dystonia, blepharospasm and hemifacial spasm-a systematic review. Toxicon. 2015;93S:S66.

20. Albanese A, Abbruzzese G, Dressler D, Duzynski W, Khatkova S, Marti MJ, et al. Practical guidance for CD management involving treatment of botulinum toxin: a consensus statement. J Neurol. 2015;262: 2201-13.

21. Sampaio C, Costa J, Ferreira JJ. Clinical comparability of marketed formulations of botulinum toxin. Mov Disord. 2004;19(Suppl 8):S129-36.

22. Wenzel R, Jones D, Borrego JA. Comparing two botulinum toxin type A formulations using manufacturers' product summaries. J Clin Pharm Ther. 2007;32(4):387-402.

23. Bohannon RW, Smith MB. Interrater reliability of a modified Ashworth scale of muscle spasticity. Phys Ther. 1987;67(2):206-7.

24. Turner-Bowker D, Hogue SJ. Short Form 12 Health Survey (SF-12). In: Michalos AC, editor.
Encyclopedia of quality of life and well-being research. Dordrecht: Springer Netherlands; 2014. p. 5954-7.

25. Turner-Stokes L, Ashford SA, Jacinto J, Maisonobe P, Balcaitiene J, Fheodoroff K. Impact of integrated upper limb spasticity management including botulinum toxin A on patient-centred goal attainment: rationale and protocol for an international, prospective, longitudinal cohort study (ULIS-III). BMJ Open. 2016;123:S81.

26. Hefter H, Jost WH, Reissig A, Zakine B, Bakheit AM, Wissel J. Classification of posture in poststroke upper limb spasticity: a potential decision tool for botulinum toxin A treatment? Int J Rehabil Res. 2012;35(3):227-33.

27. Esquenazi A, Delgado MR, Hauser RA, Picaut P, Foster K, Lysandropoulos A, et al. Duration of Symptom relief between injections for abobotulinumtoxinA (Dysport $\left.{ }^{\circledR}\right)$ in spastic paresis and cervical dystonia: comparison of evidence from clinical studies. Front Neurol. 2020;11:576117.

28. de Sainte-Marie D, Lysandropoulos A. Botulinum neurotoxins are used at low doses in the treatment of spasticity in clinical practice: results from a market research analysis. Toxicon. 2018;156:S22. 